\title{
The linked-convergent distinction
}

\section{DAVID HITCHCOCK}

\author{
Department of Philosophy \\ McMaster University \\ Hamilton, Canada L8S 4K1 \\ hitchckd@mcmaster.ca.
}

\begin{abstract}
The linked-convergent distinction introduced by Stephen Thomas in 1977 is primarily a distinction between ways in which two or more reasons can directly support a claim, and only derivatively a distinction between types of structures, arguments, reasoning, reasons, or premisses. As with the deductive-inductive distinction, there may be no fact of the matter as to whether a given multi-premiss argument is linked or convergent.
\end{abstract}

KEYWORDS: argument structure, coordinatively compound argumentation, convergent, linked, Monroe C. Beardsley, multiple argumentation, Stephen N. Thomas, support

\section{INTRODUCTION}

Once upon a time introductory logic textbooks did not mention the linked-convergent distinction. See for example Cohen and Nagel (1934), Black (1946), and Copi (1978). Stephen Thomas was the first one to draw it, in 1977. ${ }^{1}$ Thomas took the term 'convergent' from Monroe Beardsley's earlier textbook, from which come also the terms 'divergent argument' and 'serial argument' (Beardsley, 1950, p. 19). A contrast concept was already implicit in Beardsley's recognition that a reason that "converges" along with one or more other reasons on a conclusion might itself consist internally of more than one coordinate premiss. Thomas refined Beardsley's concept of convergence, made the contrast concept explicit, coined the term 'linked' for it, and supplemented Beardsley's convention for diagramming convergent reasons with a convention for diagramming the linkage among the coordinate premisses of a multi-premiss reason. Independently of Thomas's innovation, Michael Scriven (1976, p. 42) introduced a similar distinction, with a different diagramming convention, but used the term 'balance of considerations' to describe an argument with a convergent support structure. Johnson and Blair (1977, p. 177) and Hitchcock (1983, pp. 49-52) appropriate Scriven's way of making the distinction.

The distinction appears with Thomas's labels and diagramming conventions as a topic in many introductory textbooks. See for example Freeman (1993, pp. 86-106), Ennis (1996, p. 39), LeBlanc (1998, pp. 32-36), Fisher (2001, pp. 32-38), Bailin and Battersby (2010, pp. 42-44), Govier (2010, pp. 37-39), Vaughn and MacDonald (2010, pp. 95-96), and Groarke and Tindale (2013, 115-119). Many of these textbooks explain

\footnotetext{
${ }^{1}$ He claims (1986, p. 457) to have introduced it in the 1973 edition of his Practical Reasoning in Natural Language, but I have been unable to find a copy of this textbook published before 1977, despite the claim (Thomas, 1977, p. ii) of copyright in 1973, 1974 and 1975.
} 
the distinction in one short section, with exercises on applying it, but neither mention nor use the distinction elsewhere-a sign that its inclusion has become a piece of scholasticism.

The distinction is intuitively clear. Where more than one premiss is offered in direct support of a conclusion, the premisses sometimes work together to support it and are in this sense linked, whereas at other times distinct subsets of them offer independently relevant reasons that "converge" on the conclusion. A paradigm case of linked support would be a deductively valid two-premiss argument where neither premiss by itself entails the conclusion, such as the argument:

(1) There is no life on Mars, because its atmosphere is in a stable equilibrium, which would not be the case if there were life on that planet.

A paradigm case of convergent support would be an appeal to disparate considerations or criteria in support of the attribution of some supervenient status to their common subject, such as the following argument:

(2) There should be no capital punishment. The death penalty violates human rights codes that forbid cruel and unusual punishment, cannot be reversed or compensated for if it is discovered that a person was innocent of the crime for which they were executed, is no less effective as a deterrent than the likely alternative of a long prison term, and is not needed to prevent a person convicted of a capital crime from repeating that crime.

Despite this intuitive clarity, it has turned out to be difficult to spell out theoretically when premisses are linked and when they "converge". This difficulty has given rise to several scholarly treatments of the distinction, among which Walton (1996) and Freeman (2011) stand out for making it a major focus of their books on argument structure.

In this paper I wish to make one main point: that the distinction is primarily a distinction among types of support, not among arguments, premisses, reasons or structures. Only derivatively can we apply the distinction to arguments, premisses, reasons and structures. This point seems to me to be obvious once one is made aware of it, but it seems not to have been made in the literature. It implies that the search is futile for a criterion of linkage in terms of the consequences for the strength of support of finding a premiss questionable or false (e.g. no support upon falsification, diminished type of support upon elimination, etc.). Nevertheless, I shall argue, the distinction is useful.

\section{CONVERGENCE: NOT MULTIPLICITY OF ARGUMENTS}

Initially we should be clear that the linked-convergent distinction is not a distinction between a single multi-premiss argument and multiple independent arguments. There is nothing particularly problematic about the concept of distinct arguments for a single conclusion. We have clear examples of such "piling on" of arguments, as in Aristotle's 
21 arguments in his Metaphysics against Plato's theory of forms (Aristotle, 1984 [4th century BCE], 988a1-8 and 990a34-993a10), Thomas Aquinas's five ways of proving the existence of God (Aquinas, 1913 [1269], I, Q. 2, Art. 3), and the 367 different ways of proving the Pythagorean theorem (http://www.wikihow.com/Prove-the-PythagoreanTheorem; accessed 201405 24). The appropriate response to such texts is to treat each argument by itself: identifying, analyzing, interpreting and evaluating it as if no other argument for the conclusion were in the offing.

There is however some controversy over how to combine the results of such evaluations. Pollock (1995, pp. 101-102) doubts that there is accrual of independent reasons, and assumes that the degree of justification for a conclusion supported by separate undefeated arguments is simply the maximum of the strengths of those arguments. He argues that cases adduced as evidence of accrual of independent reasons, such as the greater reliability of testimony when given independently by two witnesses than when given by just one of them, are in fact cases where the separate pieces of information function as premisses of a single argument. Selinger (2014) on the other hand takes a new argument to reduce the uncertainty left by any preceding arguments for the same conclusion, provided that the premisses of the new argument are independent of the premisses of its predecessors. On the basis of this intuition, he provides a formula for calculating the degree of acceptability conferred on a conclusion by a set of such independent arguments. The inputs to this formula are provided by a valuation function which assigns to each premiss and each inference (but not to the conclusion) degrees of acceptability ranging from 0 for complete unacceptability via $1 / 2$ for being neither acceptable nor unacceptable to 1 for complete acceptability. Let $v\left(\alpha_{i j}\right)$ be the degree of acceptability of a premiss $\alpha_{\mathrm{ij}}$ of an argument $\mathrm{j}$ with conclusion $\alpha$, and $w\left(\alpha \mid \alpha_{1 \mathrm{j}}, \ldots, \alpha_{\mathrm{nj}}\right)$ be the degree of conditional acceptability in this argument of its conclusion $\alpha$ given total acceptability of its premisses $\alpha_{1 \mathrm{j}}, \ldots, \alpha_{\mathrm{nj}}$. If the premisses of this argument are independent of one another and the product of their degrees of acceptability is greater than $1 / 2$ (meaning that the conjunction of the premisses is more acceptable than not), then the degree of acceptability $v_{\mathrm{j}}(\alpha)$ conferred on the conclusion $\alpha$ by the argument is the product $v\left(\alpha_{1 \mathrm{j}}\right) \cdot \ldots \cdot v\left(\alpha_{\mathrm{nj}}\right) \cdot w\left(\alpha_{\mathrm{j}} \mid \alpha_{1 \mathrm{j}}, \ldots, \alpha_{\mathrm{nj}}\right)$. (This formula can be adjusted to accommodate cases where the premisses of an argument are not independent of one another.) The degree of acceptability conferred on $\alpha$ by $m$ such arguments $(\mathrm{m}>1)$ with premisses independent of each other is given by the formula $v_{1}(\alpha) \oplus \ldots \oplus v_{\mathrm{m}}(\alpha)$, where $x \oplus y=2 x+2 y-2 x y-1$. Selinger's formula appears to give intuitively acceptable results. For example, according to the formula two independent proofs that each confer separately a total acceptability of 1 on a theorem confer together the same acceptability of 1 , whereas two independent arguments that each confer an acceptability of $3 / 4$ on a claim together confer an acceptability of $7 / 8$ and a new independent argument that confers an acceptability on a claim only slightly greater than $1 / 2$ raises the acceptability of this claim by a very small amount. Thus the conflict between Pollock's rejection of accrual of independent reasons and Selinger's acceptance of this sort of accrual comes down to a conflict of intuitions. It is an open question whether there is any compelling argument that would resolve the conflict.

There is also an interpretive difficulty in determining whether an additional supporting reason introduced by a bridging term like 'besides' or 'moreover' or 'further' is a new argument or merely an independently relevant part of a single argument. This 
difficulty is best resolved by applying a moderate principle of charity, according to which an ambiguous text or discourse is to be disambiguated in the way that makes it more plausible.

The difference between independently relevant reasons in a single argument and multiple arguments for the same conclusion implies, as Freeman (2011, pp. 108-113) has pointed out, that the pragma-dialectical distinction between coordinatively compound argumentation and multiple argumentation is not the same as the linked-convergent distinction. Multiple argumentation involves distinct speech act complexes, in each of which one or more arguments are advanced in an attempt to justify a point of view-as it happens, the same one in each case. Coordinatively compound argumentation involves a single complex of speech acts in which more than one premiss is used in direct support of a point of view. From the pragma-dialectical perspective, the linked-convergent distinction is a distinction within the class of coordinatively compound argumentation. Snoeck Henkemans (1992, pp. 96-99), for example, recognizes two types of coordinatively compound argumentation, cumulative and complementary, which stand to each other roughly (but not exactly) as convergent arguments stand to linked arguments.

Beardsley and Thomas may have contributed to confusion between multiple arguments for a single conclusion and multiple independently relevant reasons in a single argument. Indeed, they may themselves have conflated these two concepts. They diagram convergent reasoning with a separate arrow from each independently relevant reason to the conclusion, thus giving the visual impression that there are distinct inferences to be evaluated but no need for a comprehensive assessment of how well the reasons taken together support the conclusion. Further, Beardsley refers to convergent reasoning as involving "independent reasons"-a phrase that could easily be read to cover independent arguments as well as independently relevant reasons in a single argument. Further, since Beardsley gives only two examples of convergent structures (one an argument from sign [1950, p. 18] and the other an [intuitively linked] argument for an evaluation [p. 21]) and makes nothing of the concept in his approach to evaluating arguments, it is hard to flesh out his ambiguous definition of a convergent argument as one in which "several independent reasons support the same conclusion" (p. 19). Beardsley in fact made less and less use of the concept of convergence in subsequent editions of his textbook; in the second (1956) edition it is merely mentioned at the beginning of a check-up quiz, and it is missing from the third (1966) and fourth (1975) editions. It seems then that users of the first edition did not find its concept of convergence particularly useful. For his part, Thomas (1977, p. 39) conflates independently relevant reasons in a single argument with distinct arguments sharing a conclusion by counting as convergent reasoning not only independent reasons for some action but also separate alleged proofs of a single claim, such as different arguments for the existence of God. ${ }^{2}$

\section{THE PRIMARY SPHERE OF THE DISTINCTION}

\footnotetext{
${ }^{2}$ This example disappears from the fourth (1997) edition of his textbook. A third type of example, in which a claim is supported both by evidence and by testimony, occurs only in the first two editions $(1977,1981)$ of his textbook
} 
To get a sense of the primary field of application of the linked-convergent distinction, we need to go beyond the intuitive distinction between premisses that work together and premiss-sets that constitute independently relevant reasons. We need to look at how the distinction is used, and in particular how the concept of convergent reasoning is applied. For this purpose, our most extensive and therefore best sources are the treatment of practical decision-making in the various editions of Thomas's textbook (1977, 1981, $1986,1997)$ and the treatment of conductive reasoning in the various editions of Trudy Govier's textbook (Govier, 1985, 1988, 1992, 1997, 2001, 2005, 2010).

In the last edition of his textbook (Thomas, 1997), which presumably incorporates his most developed thinking on the topic, Thomas devotes 57 pages (385-441) to practical decision-making. He recommends a five-component approach to important personal decision-making situations:

1. Identify mutually exclusive options.

2. For each option, articulate whatever possible reasons pro and con one can think of.

3. Evaluate separately the acceptability and relevance of each such reason.

4. Consider reasons bearing on the acceptability or relevance of each reason (and reasons bearing on the acceptability or relevance of those reasons, and so on).

5. Pick the option that is best supported by its undefeated pro reasons and least opposed by its undefeated con reasons.

Diagramming these components is helpful, and perhaps even essential, for keeping track of one's reasoning. In diagramming the reasoning concerning each option, Thomas uses separate arrows for each reason-solid if it is a pro reason, dashed if it is a con reason (including a reason against the acceptability or relevance of another reason). He illustrates his recommended procedure with reference to two decision-making situations, described initially in the words of the decision-maker: a choice of living accommodation (pp. 395-404) and a choice of whether to move cities in order to get a better job in one's company (pp. 414-430).

We find a similar approach in Trudy Govier's treatment of what she calls "conductive arguments" (Govier, 2010, p. 353), which she characterizes as "arguments in which premises are put forward as separately and non-conclusively relevant to support a conclusion, against which negatively relevant considerations may also be acknowledged" (2011, p. 262) and whose structure she describes as "always convergent" (2010, p. 352). Like Thomas, she proposes that one evaluate such arguments by considering for each premiss separately not only whether it is rationally acceptable but also whether it is relevant, positively or negatively, to the conclusion. After having done so, one should judge the strength of support given by each positively relevant rationally acceptable reason separately and by these reasons cumulatively, the strength of opposition given by each negatively relevant rationally acceptable counter-consideration separately and by these counter-considerations cumulatively, and the size of the difference between the cumulative support and the cumulative opposition (Govier, 1999, p. 170; 2010, pp. 365366). Govier illustrates this complex procedure with reference to an invented argument for legalizing voluntary euthanasia (Govier, 2010, pp. 360-363).

Thomas and Govier have developed more extensively than any other authors a procedure for evaluating convergent reasoning and argument. Although their procedures differ and are illustrated by application to different types of arguments, they have an 
important commonality: separate judgment of the relevance to some conclusion of each of a number of diverse considerations, criteria, or signs. The point of distinguishing independently relevant, or putatively relevant, reasons pro and con in a convergent structure is thus to isolate them for separate consideration. If a given reason turns out to be unacceptable, questionable or irrelevant, it is still possible to estimate the strength of support that the remaining acceptable and relevant reasons give to the conclusion. The partitioning into distinct reasons is a necessary preliminary to this evaluative approach, but would generally not be helpful for evaluating other types of arguments.

The appropriate criterion for convergence, then, is the independent relevance to a conclusion of distinct sub-sets of an argument's premisses. Relevance in this sense is an ontic property, that of counting in context for or against the conclusion drawn. It is not a mental property of the person putting forward the argument, such as the arguer's intention or belief. Nor is it a property of the argumentative text, such as a claim or textual indication that the supporting reasons are being put forward as independently relevant. Convergence is thus primarily a feature of the way in which multiple coordinate premisses of a piece of reasoning or argument in fact work to support the conclusion. They do so convergently when and only when distinct sub-sets of the premisses adduce distinct considerations or criteria or signs that are in fact relevant, positively or negatively, to the conclusion drawn.

Although convergence is primarily a property of the support that multiple coordinate premisses provide to a conclusion, one can apply the concept derivatively to reasoning, arguments, premisses, reasons and argument structures. Reasoning and argument are convergent when they have multiple coordinate premisses that can be partitioned into distinct sub-sets that it is plausible to interpret as put forward as independently relevant to the conclusion. In that case, the reasoning or argument can be said to have a convergent structure. The reasons constituted by such distinct sub-sets should then be treated as being put forward as convergent, i.e. as independently relevant to the conclusion, even if on evaluation not all of them turn out to be both rationally acceptable and relevant. If any such reason consists of a single premiss, then one can take that premiss to be put forward as convergent; otherwise, the concept of convergence should not be applied to the individual premisses.

Since convergence is primarily a way that a claim can be supported, there is judgment involved in deciding to treat a piece of reasoning or argument by the procedure appropriate to a convergent support structure. In cases where the reasons into which one partitions multiple coordinate premisses are not all rationally acceptable and relevant, the decision to partition may rest on syntactical considerations (e.g. a number of premisses attributing various characteristics to a common subject to which the conclusion attributes some further characteristic), semantic considerations (e.g. the status of the conclusion as a policy decision and the corresponding status of the distinct premiss-sets as diverse consequences or rules or deontic principles, or the status of the conclusion as a diagnosis and the corresponding status of the distinct premiss-sets as diverse signs or symptoms), textual considerations (e.g. the introduction of a subsequent premiss-set by the word 'besides'), and perhaps other sorts of considerations. Decisions to partition premisses based on such considerations are not correct or incorrect, but only more or less reasonable. Thus there may be no fact of the matter about whether a particular piece of reasoning or argument with multiple coordinate premisses is convergent, since the case 
for partitioning the premisses may be about as strong as the case against partitioning them. In this respect, the situation is exactly like that of deciding whether a piece of reasoning or argument is deductive, i.e. appropriately evaluated by the standard of deductive validity. The claim of the present paper that convergence is primarily a way in which a claim can be supported rather than primarily a type of argument is exactly parallel to my claim long ago that deduction is primarily a type of validity rather than a type of argument (Hitchcock, 1979).

What about the concept of linkage? If we take linkage to be the complement of convergence, we can define it as support by multiple coordinate premisses in some way other than by distinct considerations or criteria or signs that are separately relevant, positively or negatively, to the conclusion drawn. As with convergence, we can derivatively define linked reasoning, arguments, premisses, and argument structures as those that it is appropriate to treat for evaluative purposes as linked. Judgment will be involved in making the decision about appropriateness.

This conception of linkage is purely negative. It implies nothing about the effect on the strength of support of finding that a premiss of an argument with linked support is questionable or unacceptable. And a fortiori it implies nothing about this effect in the case of an argument or reasoning that one decides, appropriately or not, to treat as linked for evaluative purposes. Thus, if we accept this conception of linkage, we should regard as exercises in futility the many attempts in the literature to find a criterion for linkage in the consequences of "suspending" a premiss or finding it false: diminished support upon falsification (Thomas, 1977, p. 38), no support upon falsification (Copi, 1982, p. 21), insufficient support upon elimination (Snoeck Henkemans, 1992), type reduction upon elimination (Vorobej, 1994), and so forth. In any case, there is a useless spinning of wheels in applying any such test if the point of classifying an argument as linked is to facilitate evaluation, since one has to do the evaluation first in order to classify the argument in a way that indicates how one is to do the evaluation. Better just to do the evaluation and forget about the classification.

It might be doubted that suspension or falsification of a premiss in an argument with linked support for the conclusion can have no effect at all on the strength of support that it gives to that conclusion. A simple example of such an argument is one that has a redundant premiss whose suspension or falsification does not affect the status of the other premisses-for example, the argument:

(3) If there were life on Mars, its atmosphere would be in an unstable equilibrium; the atmosphere on Mars is not in an unstable equilibrium; Mars is an asteroid; therefore, there is no life on Mars.

The third premiss is known to be false, but this fact does not affect the strength of support given by the argument, which is in fact conclusive, given that the first and second premisses are both known to be true.

How then should we evaluate an argument that we decide to treat as if its support were linked? A straightforward way is to judge first the status of each premiss separately, in terms for example of whether it is acceptable, questionable or unacceptable. Then determine how strongly the premisses with their attributed statuses collectively support the conclusion and whether in context that degree of support is enough. It is important in 
such an exercise not to treat a premiss found to be questionable as if it had never been part of the argument, since its questionable status might affect the strength of support differently than its omission would have. Consider for example the following argument:

(4) Since everyone would agree on reflection that public knowledge that physicians may deceive their patients about their medical status would have worse consequences than public knowledge that physicians may not so deceive their patients, then physicians should not engage in such deception, for violations of the moral rule against deception are not justified under such conditions (cf. Gert, 2005).

If one finds the major premiss questionable, then one should take the argument to provide at best weak support for the conclusion, whereas one might reasonably take a variant of the argument without the major premiss to provide moderate support for the conclusion.

\section{CONCLUSION}

The linked-convergent distinction introduced by Stephen Thomas (1977) is not the same as the distinction between a single argument for a claim and multiple arguments for a claim. It is a distinction to be applied within the class of single arguments for a claim, specifically to such arguments with more than one premiss. It is primarily a distinction between ways in which two or more premisses in such an argument can directly support a claim. Support is convergent if the premisses can be partitioned into independently relevant reasons that each consist of rationally acceptable premisses. Support is linked if the premisses cannot be partitioned into independently relevant reasons that each consist of rationally acceptable premisses. One can classify arguments, reasoning, premisses, or structures as linked or convergent only in a secondary or derivative sense, where what is involved is a judgment call on what type of support the argument, reasoning or component is attempting to provide. Hence, as with the deductive-inductive distinction, there may be no fact of the matter as to whether a given multi-premiss argument is linked or convergent.

The value of the distinction lies in the consequences of treating an argument component as having convergent structure. Such a decision introduces into the evaluation of the premisses a consideration of the independent relevance of each premiss-set that is partitioned as a reason-a step that makes no sense if one is treating it as having linked structure. We should not automatically assume, however, that we can refute an argument component that we are treating as having linked structure by refuting just one of its premisses. We need to check and see.

\section{REFERENCES}

Aquinas, T. (1913). Summa theologica, Part I, QQ. 1-XXVI. London: Burns, Oates \& Washbourne. Latin original first published in 1269. 
Aristotle. (1984). Metaphysics. In J. Barnes (Ed.), The Complete works of Aristotle: The revised Oxford translation (pp. 1552-1728). Bollingen Series 71. Princeton: Princeton University Press. Greek original written in the 4th century BCE.

Bailin, S., \& Battersby, M. (2010). Reason in the balance: An inquiry approach to critical thinking. Toronto: McGraw-Hill Ryerson.

Beardsley, M. C. (1950). Practical logic. Englewood Cliffs, NJ: Prentice-Hall.

Beardsley, M. C. (1956, 1966, 1975). Thinking straight: Principles of reasoning for readers and writers. 2nd, 3rd and 4th editions of (Beardsley, 1950). Englewood Cliffs, NJ: Prentice-Hall.

Black, M. (1946). Critical thinking. New York: Prentice-Hall.

Cohen, M. R., \& Nagel, E. (1934). An introduction to logic and scientific method. New York: Harcourt, Brace.

Copi, I. M. (1978). Introduction to logic. 5th edition. New York: Macmillan.

Copi, I. M. (1982). Introduction to logic. 6th edition. New York: Macmillan.

Ennis, R. H. (1996). Critical thinking. Upper Saddle River, NJ: Prentice-Hall.

Fisher, A. (2001). Critical thinking: An introduction. Cambridge: Cambridge University Press.

Freeman, J. B. (1993). Thinking logically: Basic concepts for reasoning, 2nd edition. Englewood Cliffs, New Jersey: Prentice Hall.

Freeman, J. B. (2011). Argument structure: Representation and theory. Argumentation Library Volume 18. Dordrecht: Springer.

Gert, B. (2005). Common morality: Deciding what to do. New York: Oxford University Press.

Govier, T. (1985, 1988, 1992, 1997, 2001, 2005, 2010). A practical study of argument, 1st through 7th editions. Belmont, CA: Wadsworth.

Govier, T. (1999). Reasoning with pros and cons: Conductive argument revisited. In T. Govier, The Philosophy of Argument (pp. 155-180). Newport News, VA: Vale Press.

Govier, T. (2011). Conductive arguments: overview of the symposium. In J. A. Blair \& R. H. Johnson (Eds.), Conductive argument: An overlooked type of defeasible reasoning (pp. 262-276). London: College Publications.

Groarke, L. A., \& Tindale, C. W. (2013). Good reasoning matters: A constructive approach to critical thinking. Don Mills, ON: Oxford University Press.

Hitchcock, D. (1979). Deductive and inductive: Types of validity, not types of argument. Informal Logic, $3(2), 9-10$.

Hitchcock, D. (1983). Critical thinking: A guide to evaluating information. Toronto: Methuen.

Johnson, R. H., \& Blair, J. A. (1977). Logical self-defense. Toronto: McGraw-Hill Ryerson.

LeBlanc, J. (1998). Thinking clearly: A guide to critical reasoning. New York: W. W. Norton.

Pollock, J. (1995). Cognitive carpentry: How to build a person. Cambridge, MA: MIT Press.

Scriven, M. (1976). Reasoning. New York: McGraw-Hill.

Selinger, M. (2014). Towards formal representation and evaluation of arguments. Argumentation, 28 (3), 379-393.

Snoeck Henkemans, A. F. (1992). Analyzing complex argumentation: The reconstruction of multiple and coordinatively compound argumentation in a critical discussion. Dissertation, University of Amsterdam.

Thomas, S. N. (1977, 1981, 1986, 1997). Practical reasoning in natural language. 1st through 4th editions. Englewood Cliffs, NJ: Prentice-Hall.

Vaughn, L., \& MacDonald, C. (2010). The power of critical thinking. 2nd Canadian edition. Don Mills, ON: Oxford University Press.

Vorobej, M. (1994). The TRUE test of linkage. Informal Logic, 16(3), 147-157.

Walton, D. (1996). Argument structure: A pragmatic theory. Toronto: University of Toronto Press. 